\title{
Perceptual latencies to discriminate surface orientation in stereopsis
}

\author{
MARK F. BRADSHAW and PAUL B. HIBBARD \\ University of Surrey, Guildford, England \\ and \\ BARBARA GILLAM \\ University of New South Wales, Kensington, New South Wales, Australia
}

\begin{abstract}
The difference in sensitivity to stereoscopic surfaces oriented horizontally or vertically (the stereoscopic orientation anisotropy) can be redescribed as a difference in sensitivity to shear or compression transformations that relate the binocular images. The present experiment was designed to test this by dissociating the image transformation from the orientation of the surface. Surfaces were presented in isolation or in the presence of a surrounding frame that formed step and gradient discontinuities in the disparity field. Without discontinuities, observers required considerably more time to discriminate between surfaces differing in compression than between those differing in shear, irrespective of surface orientation. Disparity discontinuities facilitated the perception of the disparity gradients; minimum stimulus durations were reduced by over an order of magnitude when the reference frame was present. These results support the hypothesis that the disparity field is decomposed into different primitives during the recovery of depth and surface structure.
\end{abstract}

When planar surfaces, slanted in depth, are depicted within random-dot stereograms they often take a considerable time to be perceived. Gillam and colleagues (Gillam, Chambers, \& Russo, 1988; Gillam, Flagg, \& Finlay, 1984), for example, found that some observers took over $60 \mathrm{sec}$ to report seeing a surface slanted about a vertical axis (the average time was $35 \mathrm{sec}$ ). Not only does it take time to see these surfaces, but the magnitude of slant has been shown to build-up as a function of observation time (see also Julesz, 1971). Rogers and Bradshaw (1994) found that the perceived slant of large, whole-field surfaces rotated about a vertical axis increased for presentation times of up to $30 \mathrm{sec}$, and van Ee and Erkelens (1996) reported comparable findings for surfaces slanted about both horizontal and vertical axes for presentation times up to $20 \mathrm{sec}$ (but see Frisby, Bradshaw, Buckley, \& Crawford, 1992).

The slow build-up of depth in certain situations suggests strongly that the registration of binocular disparity may represent only the first step in the resolution of depth information. The disparity map produced when correspondence between the left and the right eyes' images is achieved may form the input to further stereoscopic processes that compute depth and structure from particular properties of the disparity field, such as relative disparities, disparity

Correspondence concerning this article should be addressed to M. F. Bradshaw, Department of Psychology, School of Human Sciences, University of Surrey, Guildford GU2 5XH, England(e-mail: m.bradshaw @ surrey.ac.uk). discontinuities, whole-field disparities, and disparity ramps (e.g., Erkelens \& Collewijn, 1985; Gillam et al., 1988; Gillam et al., 1984; Howard \& Kaneko, 1994; Kaneko \& Howard, 1996; Mitchison \& Westheimer, 1984). There is now a substantial body of evidence, from a range of experimental approaches, to suggest that such aspects of the disparity field can affect the resolution of depth information from binocular disparity and its time course (e.g., Anstis, Howard, \& Rogers, 1978; Graham \& Rogers, 1982; Howard \& Kaneko, 1994; Mitchison, 1993; Mitchison \& Westheimer, 1984; Stevens \& Brookes, 1988; Tyler, 1990).

The specification of the circumstances that affect latency for stereoscopic resolution, therefore, may shed light on the nature of the underlying mechanisms. Gillam et al. (1988; Gillam et al., 1984) found that resolution latencies for disparity-defined surfaces were reduced substantially by the presence of discontinuitiesin the disparity gradients (i.e., where changes in surface orientation occur). Similarly, van Ee and Erkelens (1996) found that perceived slant built up more quickly, and to greater levels, in the presence of a neighboring frontal plane (reference) surface (see also Rogers \& Bradshaw, 1994). Gillam et al. (1988), however, showed that such a visual reference facilitates the perception of surface orientation only when it creates a gradient of relative disparity. They found that discontinuities in the disparity gradient in the direction of the gradient (corresponding to "hinge" stimuli) were not as effective as discontinuitiesin the orthogonal direction (corresponding to "twist" stimuli), because the latter are always accompanied by a gradient of relative disparity. 
Inclination
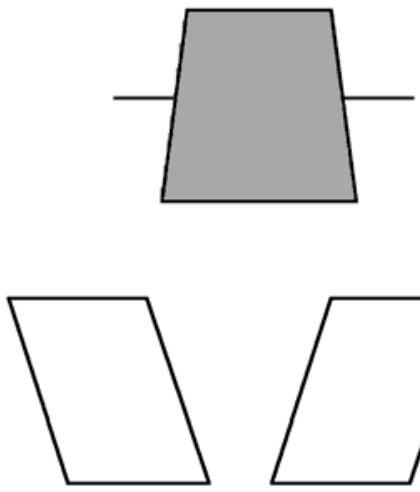

Left eye

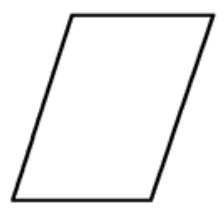

Right eye

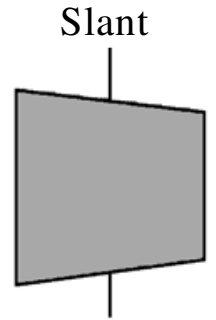

Figure 1. The left and right eyes' images generated by a surface rotated around a horizontal axis (inclination) and by a surface rotated around a vertical axis (slant). The binocular images shown in the lower panel are related by a shear (inclination) or by a compression/expansion (slant) transformation. These transformations are the basis of the stereograms shown in Figure 2.

\section{Slanted Planar Surfaces:}

\section{The Stereoscopic Anisotropy}

For slanted surfaces, the axis about which the surface is rotated is an important determinant of stereoscopic latency (see Figures 1 and 2).

Gillam et al. (1988) found a difference of $20 \mathrm{sec}$ in the mean latencies to discriminate surfaces rotated about a vertical axis (defined as slant), as compared with those for surfaces rotated about a horizontal axis (defined as inclination). The perception of inclination was faster. This difference in performance between the two orientations is one aspect of the so called stereoscopic anisotropy, which is also manifest in terms of differences in threshold performance and differences in the magnitude of perceived depth, despite the fact that the magnitudes of the positional disparities are the same in each case (e.g., Bradshaw \& Rogers, 1999; Cagenello \& Rogers, 1993; Gillam \& Rogers, 1991; Mitchison \& McKee, 1990; Parton, Bradshaw, Davies, \& Rogers, 1996; Rogers \& Graham, 1983).

The stereoscopic anisotropy could result from differences in sensitivity to the underlying binocular transformations that relate the images created when viewing surfaces rotated about vertical and horizontal axes. Figure 1 shows the image transformations that occur for these two directions of rotation. When a surface is rotated about a horizontal axis (inclination) the left and right eyes' images are related by a shear transformation. When, however, a surface is rotated about a vertical axis (slant), the left and right eyes' images are related by an expansion/compression transformation (Koenderink \& van Doorn, 1976). The stereoscopic anisotropy would result if observers were differentially sensitive to the two types of transformation. However, the difference in the underlying binocular transformations is confounded with surface orientation, and so it is difficult to assess the importance of shear/compression in the explanation of the anisotropy. The primary purpose of the present paper, therefore, is to dissociate surface orientation from the binocular transformation when testing for the anisotropy.

An orientation anisotropy has been found for surfaces defined by motion parallax that is similar to that found for
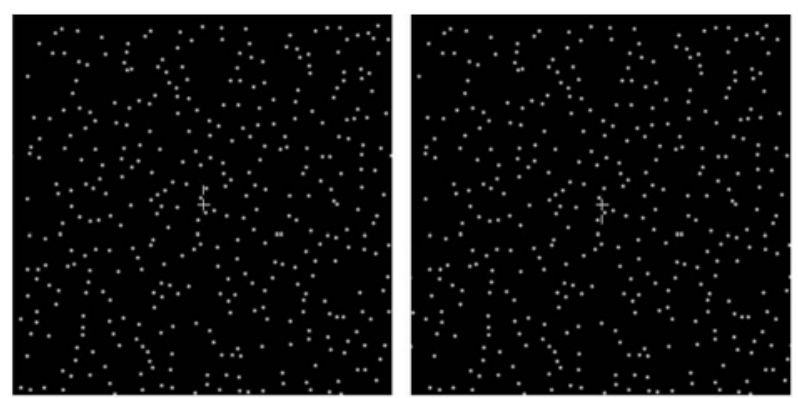

(A)
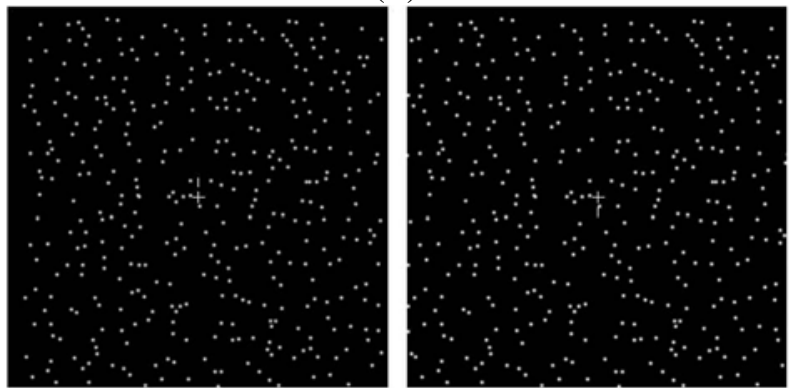

(B)

Figure 2. Examples of the slanted and inclined stimuli. The stereograms are intended for cross-eyed fusion. Panel A portrays an inclined surface, and panel $B$ portrays a slanted surface. 
binocular disparity (Rogers \& Graham, 1983; Watt, Bradshaw, \& Hogervorst, 1998). The difference in performance, in this case, can similarly be redescribed as differences in sensitivity to the underlying transformations that relate the sequence of images generated by observer or object movement. For an inclined or a slanted surface, the sequence of images generated by a horizontal head movement are related by shear and compression/expansion transformations, respectively. However, for motion, the axis of slant (i.e., slant or inclination) can be dissociated from the underlying image transformation merely by changing the direction of head or object motion. Vertical head movements create the opposite pattern of transformations for slant and inclination than that created by horizontal head movements. That is, for an inclined or a slanted surface, the sequence of images generated by a vertical head movement is related by compression/expansion or shear transformations, respectively. Making use of this fact, Rogers and Graham (1983) compared the magnitudes of the CraikO'Brien-Cornsweet depth illusion (Anstis et al., 1978) for different surface orientations and directions of head movement. They found that the magnitude of the illusion was related only to the underlying transformation, irrespective of surface orientation or the direction of head movement per se. This suggests that the anisotropy (in motion parallax) arises because the visual system is more sensitive to shear than it is to compression/expansion transformations.

Such a simple dissociation is not possible in the case of binocular disparity, since the transformation between the binocular images is generated by the (fixed) horizontal separation of the eyes in the head. However, a similar dissociation, between surface slant and image transformation, is possible if surfaces tilted in both directions around oblique $\left( \pm 45^{\circ}\right)$ axes are used (see Figure 3 ). The binocular images created by such surfaces contain both shear and compression transformations but differ in the sign of the shear or the compression component. Therefore, by determining the minimum presentation time required to discriminate between any two of the surfaces, the perceptual latency for a particular component (shear or compression) irrespective of surface orientation can be obtained. This is the major goal of the present paper.

Gillam et al. (1988) suggested that the perception of surfaces oriented around oblique axes is governed by the relative sensitivity for shear and compression disparities. They provided a demonstration stereogram (Gillam et al., 1988, p.173, Figure 12) of such a surface and argued that its perceptual resolution proceeds in two stages: Initially,
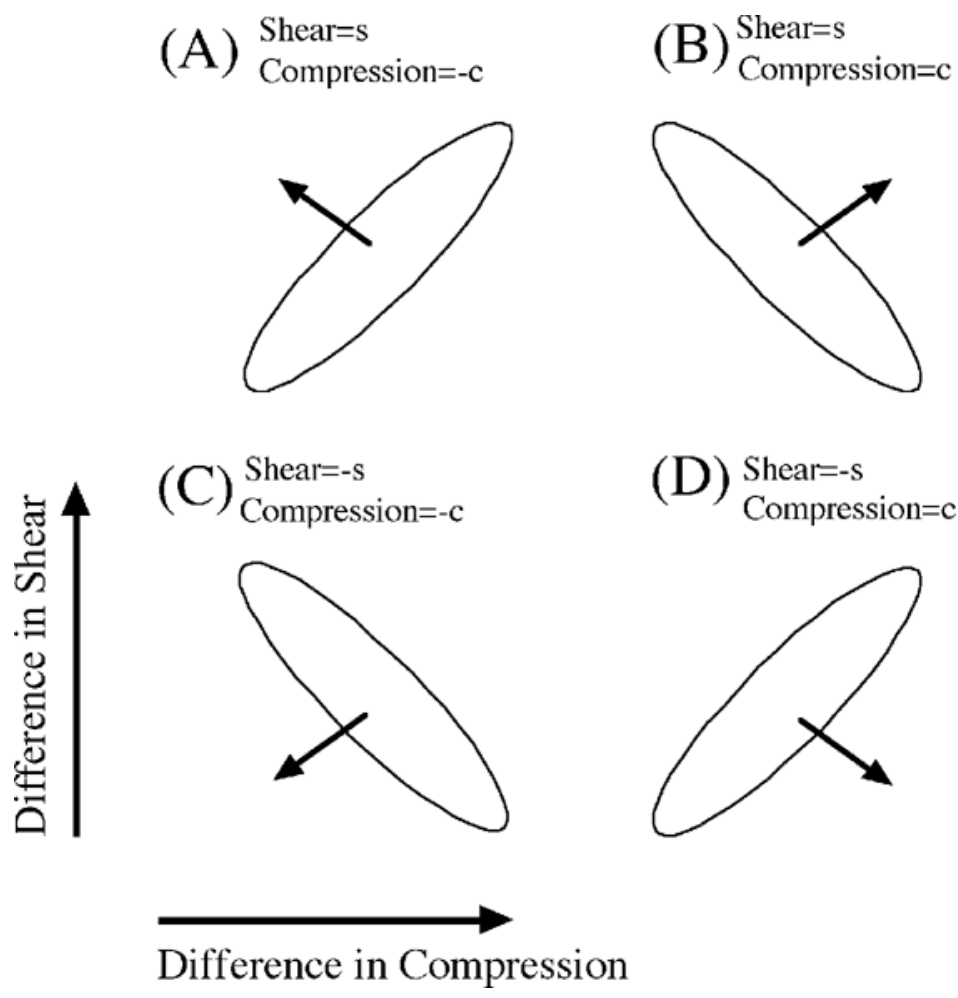

(D) Shear=-s
Compression=c

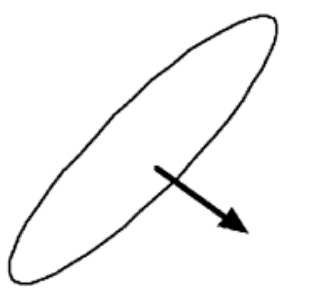

Figure 3. Representation of the surfaces that slanted about oblique $\left( \pm 45^{\circ}\right)$ axes. The legend above each pictogram describes the shear and compression disparity associated with each surface. Pairs of surfaces that are adjacent horizontally (i.e., $A B$ and $C D$ ) differ in the sign of compression disparity ( - c or c, respectively). Pairs of surfaces that are adjacent vertically (i.e., AC and BD) differ in the sign of shear disparity ( $-\mathrm{s}$ or $\mathrm{s}$, respectively). 
it appears inclined, and only later does it appear to slant about a diagonal axis. They argued that this was due to the different time courses of the responses to shear and to compression. However, they did not measure this explicitly, nor did they compare the latencies with those for simple slants and inclinations.

\section{Perceptual Latency}

Another aim of the present experiment was to determine the shortest presentation time required for observers to discriminate between pairs of planar surfaces defined by binocular disparity. To this end, we used a staircase procedure that manipulated presentation duration relative to the performance level of the observer. A binary forcedchoice task was employed to determine when depth information sufficient to discriminate between particular surfaces becomes available. This is a novel approach, since most previous studies have used perceived depth (as a function of time) or response times as the dependent measure. For example, Gillam et al. (1988) employed a task in which observers were asked to respond when they had identified 1 of 12 possible surface configurations. This strategy had the advantage that it ensured that observers saw the full surface configuration displayed. Using a different paradigm, Rogers and Bradshaw (1994) and van Ee and Erkelens (1996) charted the build-up of perceived slant or depth as a function of presentation time. Neither the slant-matching tasks employed in the latter studies nor the unlimited presentation time used by Gillam et al. (1988) were designed to determine the minimum presentation time after which depth information from disparity becomes available. However, van Ee and Erkelens (1996) found a substantial response to slant after only $100 \mathrm{msec}$ when a visual reference was present, although they did not include a backward mask to prevent further processing when stimulus presentation ended, and so it is possible that they underestimated perception times. Frisby et al. (1992) used a binary discrimination, in addition to the measurement of depth build-up, in their study of slant perception. They reported a somewhat different set of results from those discussed above, finding that, although the magnitude of slant was underestimated, it was seen at short latencies $(<1.5 \mathrm{sec})$ and did not build up. The reason for these discrepant results is not clear. The present experiment determines the minimum presentation time for the perception of surfaces rotated around the cardinal and oblique axes. Since this is the first experiment directly measuring latency for discriminating direction of stereoscopic slant, we also measured whether this was influenced by the presence of a surrounding frame providing gradients of disparity discontinuity, as well as step discontinuities

\section{Summary}

To determine whether the stereoscopic anisotropy, in terms of differences in perceptual latency, is attributable to surface orientation or whether it is attributable to the underlying shear and compression transformations between the binocularimages, we created surfaces oriented around $\pm 45^{\circ}$ that contained both shear and compression disparities of equal magnitude. This enabled us to dissociate the type of transformation to be discriminated (i.e., shear or compression) from surface orientation in an manner analogous to that of Rogers and Graham (1983) in the parallax domain. The stimuli employed were all planar surfaces rotated about oblique $\left( \pm 45^{\circ}\right)$ axes. These surfaces contained a shear disparity and a compression disparity but differed in the sign of one of these components (see Figures 3 and 4). The observers' task was to differentiate between surfaces differing either in the sign of the shear disparity or in the sign of the compression disparity with the other component of disparity remaining unaltered. This allowed us to examine latencies for the discrimination of shear and compression components of a surface, without confounding them with the orientation of the surface. Minimum presentation durations for the perception of slant and inclination were also determined. Latencies for all surfaces were determined with and without a surrounding frame.

\section{METHOD}

\section{Participants}

Four undergraduate participants took part in the experiment and received course credit for their participation. They were naive as to the purposes of the experiment, and all had normal or corrected-to-no rmal vision and stereo acuity of at least 40 arc sec (Randot Stereotests).

\section{Stimuli}

Random dots were presented within a square boundary that subtended $6^{\circ}$ of visual angle with a density of $11 \mathrm{dots} / \mathrm{deg}^{2}$. Each dot was positioned with subpixel accuracy by using a 2-D Gaussian profile, with a spatial standard deviation of 2.5 arc min, centered on the real coordinate. The effect of this was that each dot appeared as a small blob (i.e., a defocused dot) and that the depth profile of the surfaces appeared smooth and continuous. The maximum luminance of the dots was $73.0 \mathrm{~cd} / \mathrm{m}^{2}$, and the background luminance of the screen was $0.4 \mathrm{~cd} / \mathrm{m}^{2}$. To guarantee a uniform density of dots, the pseudo-randomization technique described by Gillam et al. (1988) was employed. Monocular dots were used to fill spaces left in the stimuli by the stereoscopic transformations, to eliminate the possibility that responses could be based on global shape or size differences between the two eyes' views. A nonius fixation marker was used. Examples of the stimuli are given in Figures 1 and 3. The reference condition consisted of an additional $1^{\circ}$ wide square frame of random dots with zero disparity displayed around the stimulus surface.

\section{Apparatus}

The stimuli were presented on two Apple 12-in. monochrome monitors, driven by a Macintosh 7500 and arranged in a standard Wheatstone stereoscope configuration. The monitors were viewed through two first-surface mirrors set at $\pm 45^{\circ}$ to the median plane. The viewing distance was $114 \mathrm{~cm}$, and care was taken to ensure that vergence was correct for fixation at this distance. Each pixel subtended 1 arc min. The experiment was performed in a completely darkened room, so that the only sources of illumination were the two monitors. The monitor screens were completely masked, except for an aperture (which was not visible), through which the stimuli could be seen. This reduced the ambient lighting level to the minimum possible and prevented the edges of the monitors from acting as a visual reference. 

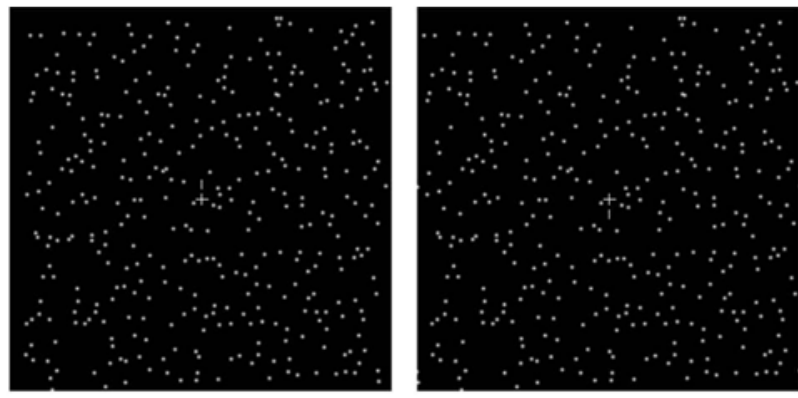

(A)
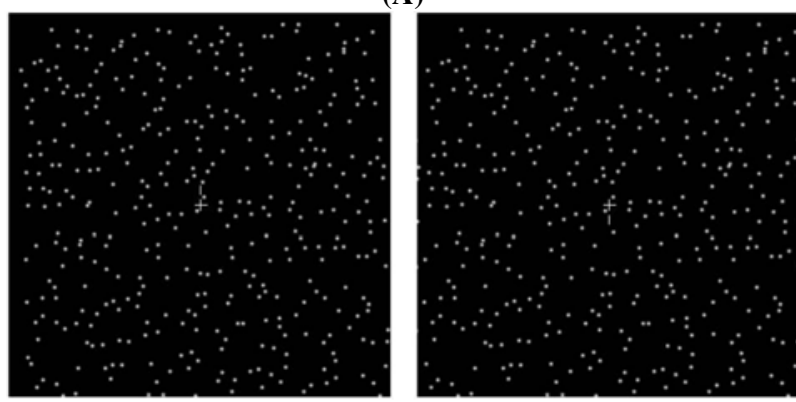

(C)
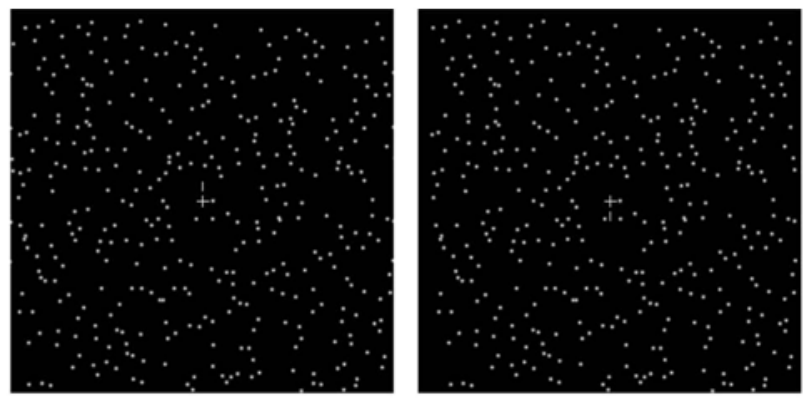

(B)
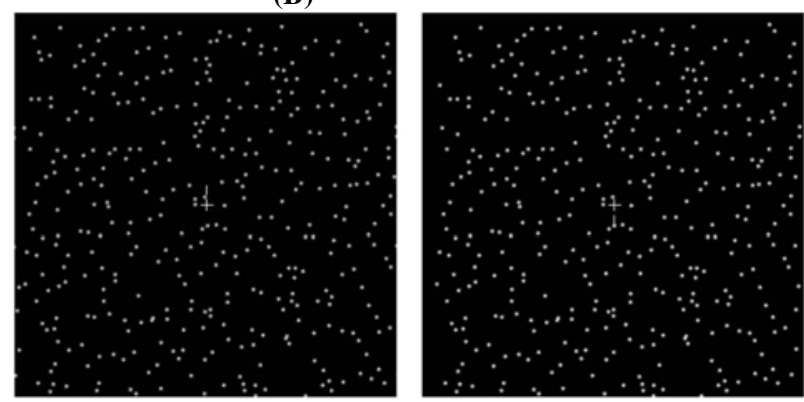

(D)

Figure 4. Examples of the stimuli that slanted about oblique $\left( \pm 45^{\circ}\right)$ axes. Stereograms are intended for cross-eyed fusion and map directly onto the four pictograms in Figure 2.

\section{Design and Procedure}

A staircase procedure (details below) was used to determine the minimum time (threshold) required to discriminate between two surfaces that differed in the sign of the shear or of the compression component (see Figure 3).

For one set of surfaces, the disparities were consistent with a surface rotated about a diagonal axis by $45^{\circ}$. Thresholds were measured independently for each of the four pairings of surfaces shown in Figures 3 and 4 . Of the two pairings in which the sign of the compression disparity was altered $(\mathrm{AB}$ and $\mathrm{CD})$, surfaces $\mathrm{A}$ and $\mathrm{B}$ differ in whether the top left or the top right corner appears farther away in depth, whereas surfaces C and D differ in whether the bottom left or the bottom right corner appears farther away. Similarly, of the pairings in which the sign of shear disparity was altered (AC and BD), $\mathrm{A}$ and $\mathrm{C}$ differ in whether the top left or the bottom left corner appears farther away, whereas B and D differ in whether the top right or the bottom right corner appears farther away.

The other set of stereograms depicted surfaces rotated about the cardinal (horizontal or vertical) axes by $\pm 45^{\circ}$ and $\pm 34.5^{\circ}$ (see Figure 2). The observers had to discriminate between the different directions of slant or the different directions of inclination in separate runs. The value of $45^{\circ}$ was the same as that used by Gillam et al. (1988) and Frisby et al. (1992), and $34.5^{\circ}$ was used since it created the same magnitude of shear and compression as that created in the surfaces oriented around oblique axes at $45^{\circ}$. The two magnitudes were randomly interleaved in a particular experimental block, and separate thresholds were tracked for each.

At the start of each block of trials, the observers were shown the two surfaces that would appear. On each trial, the stimulus was presented for a fixed time and then replaced by a random disparity mask. A similar mask has been employed previously (Bradshaw, Rogers, \& DeBruyn, 1995) and served the important function of disrupting further processing of stimulus disparity. It was important to ensure that the time available to the observers to process disparity information was of a definite duration, since Uttal, Davis, and Welke (1994) have shown that stereopsis may proceed on the basis of images presented for as little as $1 \mathrm{msec}$ when no mask is used. The mask was a randomdot stereogram, similar to the test stimuli, except that each dot was randomly assigned a crossed or uncrossed disparity of between 0 and 10 arc min. The mask appeared as a cloud of dots, occupying a three-dimensional volume of space.

The procedure started at a presentation duration at which the surfaces were clearly discriminable. After three successive correct responses, the duration was reduced by $20 \%$, and after one wrong response, it was increased by $20 \%$. The experimental run ended after 10 reversals had occurred. The mean of the last six reversal points was taken as the $79 \%$ threshold perception time. The final threshold was the mean of three estimates obtained with this procedure.

\section{RESULTS}

No significant difference was found between the surface pairs that differed in either the sign of the shear (AC and $\mathrm{BD}$ ) or the sign of the compression (AB and $\mathrm{CD}$ ), and so the results were averaged and presented together. Figure 5 presents the minimum duration times for the surfaces with no reference. The plot shows the results of discriminating the sign of the shear or the compression transformation presented in isolation (i.e., inclined or slanted surfaces, left bars) or in combination (i.e., surfaces oriented about $\pm 45^{\circ}$ axes, right bars). On average, the minimum duration times were quite long. Inclined surfaces were discriminated after $4 \mathrm{sec}$, and slanted surfaces took around $2 \mathrm{sec}$ longer. This is another instance of the stereoscopic orientational anisotropy. 
The discrimination of the shear component in the oblique surfaces took just over $1 \mathrm{sec}$ and was clearly faster than the discrimination of the compression component $(6 \mathrm{sec})$, which was of a similar order as the response to slant $(6.2 \mathrm{sec})$. The perception of shear, therefore, seems to be facilitated by the presence of compression, although no reverse facilitation (i.e., the presence of shear affecting the perception of compression) was evident.

Figure 6 presents the minimum duration times for the surfaces with a visual reference. Clearly, the addition of a reference surface has a substantial effect on the absolute duration thresholds, which fell by at least an order of magnitude in all cases. Response latencies for the oblique surfaces and the inclination were around $100 \mathrm{msec}$, whereas for slant they were slightly longer $(220 \mathrm{msec})$. Although the stereoscopic anisotropy was reflected in different latencies for inclination and slant, the advantage of shear over compression in the oblique surfaces was negated with the addition of the reference. This may have been due to a floor effect.

The results were analyzed using a three-way repeated measures analysis of variance (log transforms), with rotation axes (cardinal or oblique), disparity component (shear or compression), and reference (present or absent) as factors. All the main effects were significant. That is, oblique surfaces were detected faster than cardinal surfaces $[F(1,10)=$ $6.3, p<.05]$, the shear component was detected faster than compression $[F(1,10)=14.2, p<.01]$, and the reference reduced all latencies significantly $[F(1,10)=171.1, p<$ $.0001]$. The fact that oblique surfaces were resolved faster than those tilted around the cardinal axes suggests that the presence of the additional disparity component affects the processing time. That is, the two components of disparity in the oblique surfaces are not independent. The Tukey HSD test revealed that inclination was perceived significantly faster than slant $(p<.05)$ and, of particular importance, in the oblique surfaces the shear component was perceived significantly faster than the compression $(p<$ .005). Overall, the significant difference in response latency for the detection of the shear (the stereoscopic anisotropy) was not evident when the surfaces were presented within a visual reference, although the effect did persist for the surfaces rotated about the cardinal axes $(p<$ $.05)$.

\section{DISCUSSION}

The experiment reported here determined the minimum presentation durations required to discriminate the direction of tilt of two disparity-defined surfaces. The results are important, since they shed light on the stereoscopic mechanisms involved in the recovery of depth and structure from binoculardisparity information. We found that the time taken to discriminate between two surfaces that differed in the sign of the shear component was much less than the time taken to discriminate between surfaces that differed in the sign of the compression. This finding held for both inclinations and slants (where the binocular images are related by a shear or a compression) and for surfaces rotated about axes set at $\pm 45^{\circ}$ (where the binocular images are related by a shear and a compression). Therefore, we can conclude that the response to binocular image shear is faster than the response to image compression, irrespective of the orientation of the surface. This is an im-

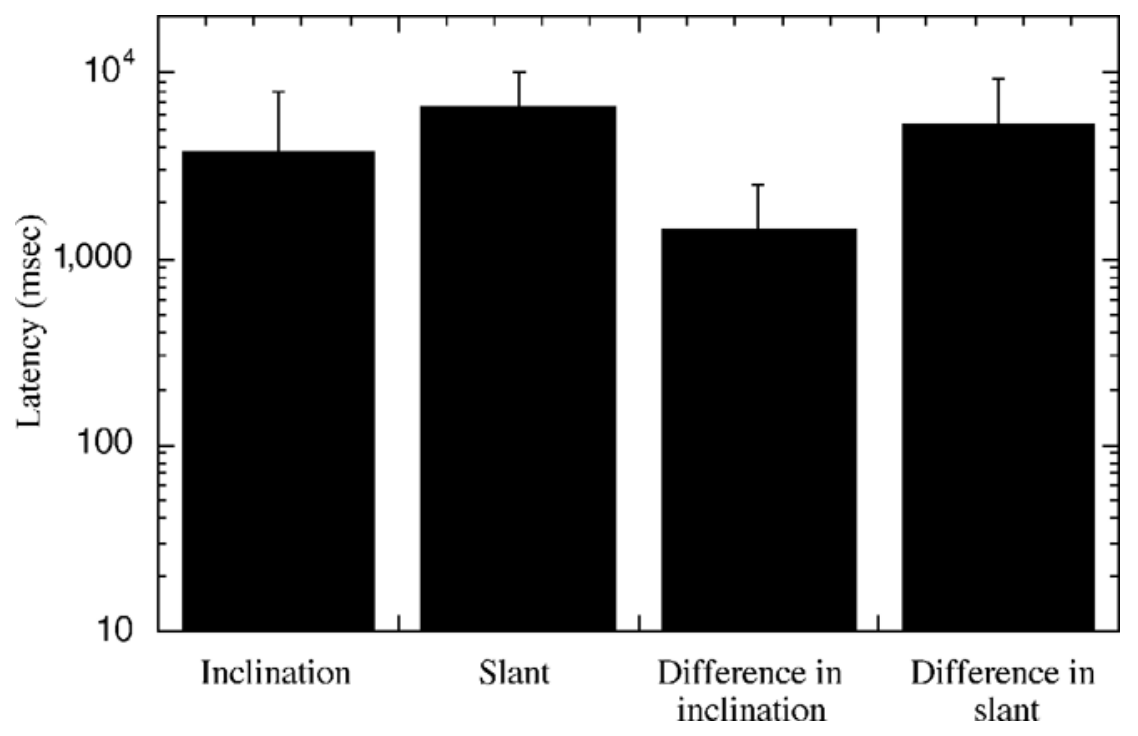

Figure 5. Latencies for the four surface discrimination tasks used for surfaces presented without a surrounding reference frame. Mean latencies across 4 observers are presented for discrimination between surface inclinations between surface slants, and between oblique surfaces differing in a component of inclination or slant. Error bars represent $+1 S D$. 


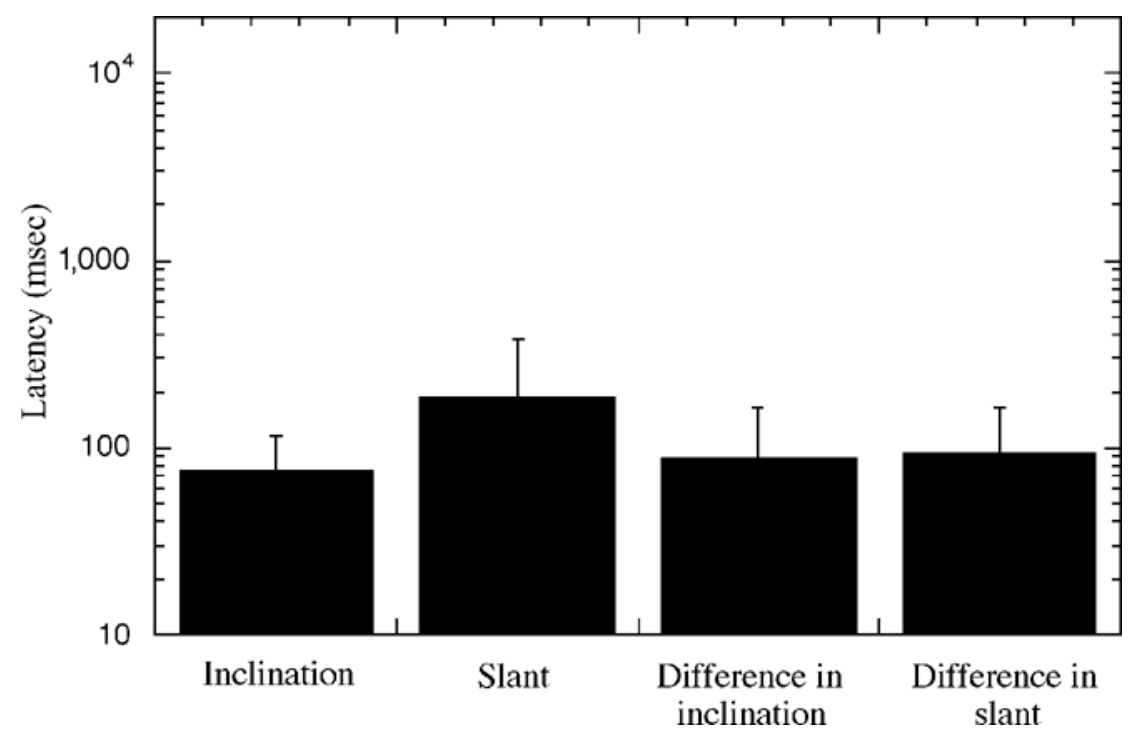

Figure 6. Latencies for the four surface discrimination tasks. The figure is similar to Figure 5 but shows latencies for the discrimination of surfaces presented with a surrounding reference frame. Error bars represent $+1 S D$.

portant result and one that concurs with the conclusions reached by Rogers and Graham (1983), who reported a similar finding in the motion parallax domain.

The stereoscopic anisotropy can, therefore, be attributed unambiguously to the differences in sensitivity to shear and compression binocular transformations. This supports the suggestion that the disparity field is decomposed into shear and compression components during the depth recovery process (Gillam et al., 1988; Koenderink \& van Doorn, 1976). Shear and compression may form different primitives for stereopsis, and each component may be processed by different mechanisms (Gillam et al., 1988; Howard \& Kaneko, 1994; Kaneko \& Howard, 1996; van Ee \& Erkelens,1995), although the fact that we found that the surfaces rotated around diagonal axes, where both transformations exist, were resolved faster than those rotated around the cardinal axes suggests that the processes that extract shear and compression are not completely independent (or may be subject to a kind of probabilistic combination at some level in the system). This type of facilitation is consistent with the results of Parton et al. (1996), who found, in a depth-matching paradigm, that disparity-defined corrugations, oriented at $45^{\circ}$, appear deeper than either their horizontal or their vertical counterparts (matched for disparity).

Although the presence of one gradient affected the perception of the other in the oblique surfaces, the magnitude of the effect was small relative to the effect of the surrounding frame, which reduced latencies by over an order of magnitude. The surrounding frame introduced gradients of disparity discontinuity, as well as step discontinuities in disparity, which may be important for the resolution of depth from stereopsis. In addition to changes in the local aspects of the disparity field, changes in the global characteristics may also be important. For example, the spatial frequency content (considered in the disparity domain) of the stimuli is changed when discontinuities are present, with more power in moderate and high frequencies than in stimuli depicting simple planar surfaces. This may be important, since the visual system is most sensitive to depth modulations of around 0.4 cycles per degree (Bradshaw \& Rogers, 1999; Rogers \& Graham, 1982; Tyler, 1974) and its sensitivity falls off when only higher or lower frequencies are present. Although parsimonious, since it accounts for the results with a single mechanism based on the spatial integration of disparity, whether such a model based on threshold vision applies to the perception of suprathreshold surfaces is uncertain (see Ioannou, Rogers, Bradshaw, \& Glennerster, 1993).

Alternatively, the disparity discontinuities created by the frame allow the interpretation of the disparity field to proceed without the need for extraretinal signals of head and/or eye position. Disparity gradients created by planar slanted or inclined surfaces, viewed in the absence of any visual reference, are ambiguous cues for slant, since they may have been created by head rotations about a horizontal or a vertical axis (see, e.g., Backus, Banks, van Ee, \& Crowell, 1999; Rogers \& Bradshaw, 1994; van Ee \& Erkelens, 1996). The addition of a visual reference, which reduces this ambiguity, may be sufficient to decrease perceptual latency. Similarly, the fact that no simple pattern of head or eye movements can create the pattern of shear and compression disparities generated by surfaces rotated about $\pm 45^{\circ}$ may have decreased the perceptual latency for these surfaces. Another interpretation of why adjacent reference surfaces affect perception was put forward by 
Howard and Rogers (1995), who suggested that the percept of single disparity gradients may default (or normalize) to the frontal plane through the equidistance tendency (Gogel, 1956). Mitchison and Westheimer (1984) argued that the spatial configuration of disparity is crucial for depth perception. For a range of stimuli, they showed that perceived depth was a function of the summed disparities, weighted according to the proximity, between test objects and their neighbors. In other words, perceived depth from a particular disparity is determined relative to a local norm based on the average local disparity values (i.e., its salience).

The absolute perception times are also of interest. Even with the simplicity of the task in the present experiment, the time taken to resolve slanted surfaces was, on average, $6 \mathrm{sec}$, with some observers taking over $10 \mathrm{sec}$. The classification of inclination was completed faster but still took, on average, $3 \mathrm{sec}$. Of course, the discrimination of disparity gradients presented in complete isolation is something that would occur only very rarely within the real world, and the experimental stimuli should, therefore, be viewed as psychophysical tools used to reveal the underlying mechanisms involved in slant perception. More representative of real-world scenes, although still far removed from them, are the stimuli that contained disparity discontinuities created by the frame. Of course, even when stimuli do comprise such discontinuities, the disparity gradients must still be processed to enable judgments of surface orientation. With the presence of discontinuities, threshold stimulus durations for slant discrimination fell to less than $250 \mathrm{msec}$ in all conditions. In absolute terms, the latencies in both conditions are much lower than those reported by Gillam et al. (1988), which is to be expected given the many procedural differences in the design of the two studies. Foremost is that we presented stimuli for a definite duration and used a two-alternative forced-choice task, whereas Gillam et al. (1988) measured response with a 12-alternative forced-choice task, which may have shifted the criterion used. Certainly, the task used in the present study could be performed by using considerably less information (the direction of the disparity gradient) than that required for the task used by Gillam et al. (the discrimination of disparity gradients and their spatial configuration). The task employed in the present study is also different from the slant-matching procedures used by other researchers (Gillam et al., 1984; Rogers \& Bradshaw, 1994; van Ee \& Erkelens, 1996). In these studies, it was found that the magnitude of perceived depth increases slowly over time. The present study augments this picture by showing that it takes a definite (and often extended) period of time to access this information initially and that this time period differs for the perception of slant and inclination (Hegarty, Hibbard, Bradshaw, \& Parton, 1997; White \& Odom, 1985). Absolute latencies are mitigated substantially by the presence of disparity discontinuities, although the difference with surface orientation remains.

The results of the present experiment suggest that the stereoscopic response to shear and compression is slow (in the order of several seconds) when other reference sur- faces are not present. By dissociating the possible effects of surface orientation from the underlying binocular transformations, these results support the suggestion that compression and shear disparities are fundamental components in the recovery of depth information from disparity.

\section{REFERENCES}

Anstis, S. M., Howard I. P., \& Rogers B. J. (1978). A Craik-O'BrienCornsweet illusion for visual depth. Vision Research, 18, 213-217

Backus, B. T., Banks, M. S., van Ee, R., \& Crowell, J. A. (1999). Horizontal and vertical disparity, eye position, and stereoscopic slant perception. Vision Research, 39, 1143-1170.

Bradshaw, M. F., \& Rogers, B. J. (1999). Sensitivity to horizontal and vertical corrugations defined by binocular disparity. Vision Research, 39, 3049-3056.

Bradshaw, M. F., Rogers, B. J., \& DeBruyn, B. (1995). Perceptual latency and complex random-dot stereograms. Perception, 24, 749-759.

Cagenello, R., \& Rogers, B. J. (1993). Anisotropies in the perception of stereoscopic surfaces: The role of orientation disparity. Vision Research, 33, 2189-2201.

Erkelens, C. J., \& CollewiJn, H. (1985). Motion perception during dichoptic viewing of moving random-dot stereograms. Vision Research, 25, 583-588.

Frisby, J. P., Bradshaw, M. F., Buckley, D., \& Crawford, M. (1992). Perceptual latencies in the perception of surface slant in stereograms and real surfaces [Abstract]. Perception, 21, 17.

Gillam, B., Chambers, D., \& Russo, T. (1988). Postfusional latency in stereoscopic slant perception and the primitives of stereopsis. Journal of Experimental Psychology: Human Perception \& Performance, 14, 163-175.

Gillam, B., Flagg, T., \& Finlay, D. (1984). Evidence for disparity change as the primary stimulus for stereoscopic processing. Perception \& Psychophysics, 36, 559-564.

Gillam, B., \& Rogers, B. (1991). Orientation disparity, deformation, and stereoscopic slant perception. Perception, 20, 441-448.

Gogel, W. C. (1956). The tendency to see objects as equidistant and its inverse relation to lateral separation. Psychological Monographs, 70 (Whole No. 411).

Graham, M. E., \& Rogers, B. J. (1982). Simultaneous and successive contrast effects in the perception of depth from motion-parallax and stereoscopic information. Perception, 11, 247-262.

Hegarty, M. R., Hibbard, P. B., Bradshaw, M. F., \& Parton, A. P. (1997). Anisotropic temporal integration in the perception of stereoscopic corrugations, Perception, 26(Suppl.), 27.

HowARD, I. P., \& KANEKO, H. (1994). Relative shear disparities and the perception of surface inclination. Vision Research, 34, 2505-2517.

Howard, I. P., \& Rogers, B. J. (1995). Binocularvision and stereopsis. New York: Oxford University Press.

Ioannou, G. L., Rogers, B. J., Bradshaw, M. F., \& Glennerster, A. (1993). Threshold and supra-threshold sensitivity functions for stereoscopic surfaces [Abstract]. Investigative Ophthalmology\& Visual Science, 34, 1438.

Julesz, B. (1971). Foundations of cyclopean perception. Chicago: University of Chicago Press.

KaneKo, H., \& Howard, I. P. (1996). Relative size disparities and the perception of surface slant. Vision Research, 36, 1919-1930.

Koenderink, J. J., \& VAN Doorn, A. (1976). Geometry of binocular vision and a model of stereopsis. Biological Cybernetics, 21, 29-35.

Mitchison, G. J. (1993). The neural representation of stereoscopic depth contrast. Perception, 22, 1415-1426.

Mitchison, G. J., \& McKeE, S. P. (1990). Mechanisms underlying the anisotropy of stereoscopic tilt perception. Vision Research, 30, 17811791.

Mitchison, G. J., \& Westheimer, G. (1984). The perception of depth in simple figures. Vision Research, 24, 1063-1073.

Parton, A. D., Bradshaw, M. F., Davies, I. R. L., \& Rogers, B. J. (1996). The effect of surface orientation on the perception of stereoscopic corrugations. Perception, 25, 67-68. 
Rogers, B. J., \& BradshaW, M. F. (1994). Is dif-frequency a stimulus for stereoscopic slant? [Abstract] Investigative Ophthalmology \& Visual Science, 35, 1316.

Rogers, B. J., \& Graham, M. E. (1982). Similarities between motion parallax and stereopsis in human depth perception. Vision Research, 22, 261-270.

Rogers, B. J., \& Graham, M. E. (1983). Anisotropies in the perception of three-dimensional surfaces, Science, 221, 1409-1411.

Stevens, K. A., \& Brookes, A. (1988). Integrating stereopsis with monocular interpretations of planar surfaces. Vision Research, 28, 371-386.

TYLer, C. W. (1974). Depth perception in disparity gratings. Nature, 251, 140-142.

TYler, C. W. (1990). A stereoscopic view of visual processing streams. Vision Research, 30, 1877-1895.
Uttal, W. R., Davis, N. S., \& Welke, C. (1994). Stereoscopic perception with brief exposures. Perception \& Psychophysics, 56, 599-604.

van Ee, R., \& ERKelens, C. J. (1995). Binocular perception of slant about oblique axes relative to a visual frame of reference. Perception, 24, 299-314.

van Ee, R, \& ERKelens, C. J. (1996). Temporal aspects of binocular slant perception. Vision Research, 36, 43-51.

Watt, S. J., Bradshaw, M. F., \& Hogervorst, M. A. (1998). The effect of surface orientation on the perception of parallax-defined corrugations [Abstract]. Perception, 27, 117.

White, K. D., \& Odom, J. V. (1985). Temporal integration in global stereopsis. Perception \& Psychophysics, 37, 139-144.

(Manuscript received November 30, 1999; revision accepted for publication April 1, 2001.) 